\title{
Influência da impedância e relutância em recursos eletroterapêuticos utilizados em tratamentos fisioterapêuticos
}

\author{
Influence of impedance and reluctance in electrotherapeutic \\ resources used to physiotherapy treatments
}

Patrik Nepomuceno, ${ }^{1}$ Claudia Betina Reis, ${ }^{1}$ Luciana Cezimbra Weis ${ }^{2}$

1 Universidade de Santa Cruz do Sul (Unisc), Santa Cruz do Sul, RS, Brasil.

2 Centro Universitário Franciscano (Unifra), Santa Maria, RS, Brasil.

\section{RESUMO}

A eletroterapia consiste no tratamento através de correntes elétricas, ocasionando alterações fisiológicas. Dentro desta terapêutica temos os conceitos de impedância e relutância, temas importantes que devem ser do conhecimento do fisioterapeuta. Objetivo: analisar em dados da literatura aspectos relevantes da impedância e relutância de uma corrente elétrica, nos recursos terapêuticos. Método: foram buscados artigos científicos através de bases de dados, no período de março a junho de 2016, utilizando-se palavras-chave como "impedância", "relutância", "eletroterapia" e "fisioterapia" e suas traduções para o inglês. Resultados: nessa perspectiva encontraram-se, nesse período, sete artigos científicos, que tratavam sobre o assunto impedância, e não foram encontrados artigos científicos sobre a relutância. Considerações finais: diversos mecanismos estão relacionados à impedância da pele e sua redução está associada à sensação agradável relatada pelo paciente e à efetividade do tratamento. Também é necessário ressaltar a importância para o profissional de conhecer os assuntos tratados no presente estudo, pois assim ele poderá aplicar de maneira mais efetiva as correntes excitomotoras, gerando assim uma maior agilidade na terapêutica.

Palavras-chave: Impedância; Eletroterapia; Fisio-

\section{ABSTRACT}

Electrotherapy consists on the treatment by electrical currents that induce to physiological changes. In this therapy we have the concepts of impedance and reluctance that are important issues to be aware of the physical therapist. Objective: to analyze in scientific literature data aspects relevant to the impedance and reluctance of an electric current in the therapeutic resources. Method: scientific papers were searched in web databases from March to June 2016. We used keywords such as "impedance", "reluctantly", "electrotherapy" and "physiotherapy" and their respective translation for Portuguese. Results: we found seven scientific articles about the subject impedance in this period, and we didn't found any scientific articles about the reluctance. Closing remarks: several mechanisms are related to the impedance of the skin and its reduction is associated with pleasant sensation reported by the patient and the effectiveness of treatment. It is also necessary to emphasize the importance for the professional know the issues in this study, then he can apply more effectively the currents, thus generating greater agility in therapy.

Keywords: Impedance; Electric Stimulation Therapy; Physical Therapy Specialty. terapia. 


\section{INTRODUÇÃO}

A eletroterapia pode ser definida como o tratamento de pacientes, através da utilização de meios elétricos; assim, as correntes elétricas são aplicadas ao corpo, ocasionando alterações fisiológicas. ${ }^{1}$ As primeiras observações sobre a utilização de eletricidade para tratamento foram feitas no século 18 e, desde então, houve uma grande evolução nesta área, sobretudo nos séculos 20 e $21 .{ }^{2}$ Atualmente, a eletroterapia tem sido utilizada nas mais diversas áreas da reabilitação, como pacientes neurológicos, ${ }^{3,4}$ tratamento de feridas, ${ }^{5,6}$ incontinência urinária, ${ }^{7}$ entre outros. ${ }^{8}$

Os tecidos do corpo possuem cargas elétricas e os estímulos dentro deles ocorrem pelo movimento de íons; ${ }^{1}$ assim, o tecido estimulado deve responder à corrente elétrica de forma similar ao que funciona normalmente, para que haja efeitos terapêuticos. ${ }^{9} \mathrm{Na}$ eletroterapia existem propriedades que podem impedir ou dificultar a passagem destas correntes, por isso é necessário entender melhor os conceitos de impedância e relutância, visto que muitos profissionais ainda não possuem um entendimento claro. ${ }^{10}$

A impedância é a resistência de um circuito à uma corrente, que depende da natureza dos tecidos biológicos e da frequência da estimulação. Como fatores de impedância, podemos citar: excesso de pelos, pele seca, ossos, unhas, entre outros. ${ }^{10}$ Já, a relutância é definida como a oposição ou dificuldade que um material oferece à passagem de um campo eletromagnético, que depende do material do objeto e de suas dimensões, ${ }^{11}$ dentre esses opositores, podemos citar a gordura. ${ }^{10}$

Assim, é importante que o fisioterapeuta possua conhecimento sobre as propriedades relacionadas à eletroterapia, portanto este trabalho tem como objetivo analisar em dados da literatura, aspectos relevantes à impedância e relutância de uma corrente elétrica nos recursos terapêuticos.

\section{MÉTODO}

Para a realização dessa revisão, os artigos científicos foram obtidos por meio das bases de dados BIREME, ScieELO, Portal de Periódicos da CAPES, PubMed e LILACS, no período de março a junho de 2016. Foram buscados artigos, publicados a partir de 2005, preferencialmente, em língua inglesa ou portuguesa. Para isso, utilizou-se palavras-chave como "impedância", "relutância", "eletroterapia" e "fisioterapia", combinadas de diversas formas, sendo que estas estavam contidas nos Descritores em Ciências da Saúde - DECS. Os critérios de inclusão foram artigos que, no seu desenvolvimento, apresentassem a temática impedância e/ou relutância, relacionados à área de fisioterapia, tanto em aspectos de reabilitação, como em estética, que apresentassem metodologia clara e concisa, que contivessem resultados e conclusões pertinentes à prática profissional do fisioterapeuta. Tendo como critério de exclusão, artigos publicados em revistas não indexadas e que não cumprissem os critérios de inclusão.

\section{RESULTADOS}

Nessa perspectiva, encontraram-se 18 artigos e após a aplicação dos critérios de inclusão e exclusão foram selecionados 7 artigos que tratavam sobre o assunto impedância. Não foram encontrados artigos científicos sobre a relutância, o que gerou uma limitação na obtenção de resultados sobre os temas. A tabela 1 apresenta os artigos incluídos.

Tabela 1 - Artigos incluídos na revisão.

\begin{tabular}{|c|c|c|c|}
\hline Autores & Título do Artigo & Metodologia & Conclusão \\
\hline $\begin{array}{l}\text { Macedo, A C. } \\
\text { B; et al. }{ }^{22}\end{array}$ & $\begin{array}{l}\text { Efeitos da Aplicação da Corrente } \\
\text { Polarizada e da lontoforese na Gordura } \\
\text { Localizada em Mulheres }\end{array}$ & $\begin{array}{l}\text { Vinte e quatro voluntárias e foram aplicadas cor- } \\
\text { rentes galvânica, difásica, curto período, longo } \\
\text { período associadas à iontoforese. }\end{array}$ & $\begin{array}{l}\text { A associação da iontoforese e corrente } \\
\text { polarizada proporcionou redução da } \\
\text { medida em flancos. }\end{array}$ \\
\hline $\begin{array}{l}\text { Morais, J. D.; } \\
\text { et al. }{ }^{14}\end{array}$ & $\begin{array}{l}\text { Resistência Elétrica de Géis Lubri- } \\
\text { ficantes Utilizados na Reabilitação } \\
\text { Uroginecológica }\end{array}$ & $\begin{array}{l}\text { Este estudo analisou géis lubrificantes a base de } \\
\text { água e gel condutor para uso em eletroterapia } \\
\text { utilizados na reabilitação uroginecológica. }\end{array}$ & $\begin{array}{l}\text { Os géis de acoplamento apresentaram } \\
\text { menor resistência que a maioria dos } \\
\text { lubrificantes íntimos. }\end{array}$ \\
\hline $\begin{array}{l}\text { Morais, J. D.; } \\
\text { et al. }{ }^{15}\end{array}$ & $\begin{array}{l}\text { Géis Lubrificantes São Bons Agentes } \\
\text { de Acoplamento para Estimulação } \\
\text { Transvaginal do Assoalho Pélvico }\end{array}$ & $\begin{array}{l}\text { Estudo que analisou géis lubrificantes passíveis } \\
\text { de serem utilizados na introdução intracavitária e } \\
\text { gel condutor. }\end{array}$ & $\begin{array}{l}\text { Os géis utilizados na eletroterapia foram } \\
\text { a melhor opção pois apresentaram maior } \\
\text { condução da corrente elétrica em relação } \\
\text { aos géis lubrificantes. }\end{array}$ \\
\hline $\begin{array}{l}\text { Bolfe, V. J.; } \\
\text { Guirro, R. R. J. }\end{array}$ & $\begin{array}{l}\text { Resistência Elétrica dos Géis e Líquidos } \\
\text { Utilizados em Eletroterapia no Acopla- } \\
\text { mento Eletrodo-pele }\end{array}$ & $\begin{array}{l}\text { Análise de } 10 \text { agentes de acoplamento utilizados } \\
\text { na eletroterapia. Durante a avaliação da tensão } \\
\text { elétrica foi emitida corrente bifásica seguida de } \\
\text { corrente contínua. }\end{array}$ & $\begin{array}{l}\text { Os géis, a água potável e o soro fisiológi- } \\
\text { co foram indicados para a eletroterapia, } \\
\text { pois mantiveram baixa resistência, porém } \\
\text { a água desionizada não foi recomendada } \\
\text { pois apresentou alta resistência. }\end{array}$ \\
\hline $\begin{array}{l}\text { Roriz, I. M. } \\
\text { M.; et al. }{ }^{20}\end{array}$ & $\begin{array}{l}\text { Estimulação Elétrica Transcutânea na } \\
\text { Modulação da Dor dos Tender Points na } \\
\text { Síndrome de Fibromialgia }\end{array}$ & $\begin{array}{l}\text { Estudo de caso com paciente fibromiálgica, } \\
\text { utilização de TENS convencional como eletrodi- } \\
\text { agnóstico para mapeamento dos tender points e } \\
\text { TENS acupuntura para tratamento da dor. }\end{array}$ & $\begin{array}{l}\text { TENS mostrou-se eficiente como eletro- } \\
\text { diagnóstico dos tender points e ajudou a } \\
\text { diminuir a dor, devendo ser utilizada de } \\
\text { forma complementar. }\end{array}$ \\
\hline $\begin{array}{l}\text { Bolfe, V. J.; } \\
\text { et al. }{ }^{12}\end{array}$ & $\begin{array}{l}\text { Comportamento da Impedância Elétrica } \\
\text { dos Tecidos Biológicos durante a } \\
\text { estimulação elétrica transcutânea }\end{array}$ & $\begin{array}{l}\text { Aplicaram-se correntes de baixa e média } \\
\text { frequência em } 20 \text { voluntários e foi realizada } \\
\text { a mensuração da impedância elétrica em } \\
\text { diferentes locais corporais. }\end{array}$ & $\begin{array}{l}\text { A impedância dos tecidos variou de acordo } \\
\text { com a frequência da corrente e a locali- } \\
\text { zação dos eletrodos, apresentando padrão } \\
\text { não uniforme nos diferentes segmentos. }\end{array}$ \\
\hline $\begin{array}{l}\text { Gauglitz, A. C. } \\
\text { F.; et al. }{ }^{19}\end{array}$ & $\begin{array}{l}\text { Comparação da Agradabilidade na } \\
\text { Aplicação da Estimulação Elétrica } \\
\text { Nervosa Transcutânea e da Corrente } \\
\text { Interferencial }\end{array}$ & $\begin{array}{l}\text { Vinte e dois voluntários utilizaram as Correntes } \\
\text { Interferencial (IFC) e Estimulação Elétrica Ner- } \\
\text { vosa Transcutânea (TENS) e responderam a um } \\
\text { questionário para avaliar a agradabilidade. }\end{array}$ & $\begin{array}{l}\text { Concluíram que a TENS foi mais } \\
\text { agradável ao paciente quando } \\
\text { comparada a IFC. }\end{array}$ \\
\hline
\end{tabular}




\section{DISCUS5ÃO}

Bolfe ${ }^{12}$ investigou o comportamento da impedância, durante estimulação com correntes, aplicadas em diferentes locais do corpo, encontrando que a impedância dos tecidos responde, de forma diferente quando relacionada à localização e distância entre os eletrodos, bem como pela frequência da corrente. Os resultados obtidos com estimulação de baixa e média frequência, tanto em membros superiores, quanto em inferiores, foram iguais, porém na face posterior do tronco, a impedância se mostrou menor, quando comparadas aos membros.

Outro estudo realizado por Bolfe ${ }^{13}$ buscou relacionar a resistência dos géis e líquidos mais utilizados na eletroterapia. Assim, observou-se que os géis e líquidos diferiram com relação à resistência, aumentando gradativamente, quando submetido à corrente contínua. Dentre os géis utilizados todos se mostraram eficientes na transmissão da corrente elétrica. A solução fisiológica e água potável foram indicadas para umedecer esponjas, sendo que a solução fisiológica manteve sua resistência inalterada, durante a eletrólise, mostrando maior estabilidade elétrica. A água destilada ou desionizada não foi recomendada, somente sendo indicada na iontoforese, já que beneficiaria a repulsão do íon terapêutico.

Pesquisas recentes realizadas na área de uroginecologia tem buscado analisar a efetividade dos géis utilizados na eletroterapia e dos lubrificantes íntimos que podem ser utilizados na introdução de eletrodos intracavitários e concluíram que os géis utilizados em eletroterapia possuíam menor resistência à passagem da corrente, porém em caso de desconforto para o paciente, foi oferecida a possibilidade de que fossem substituídos por lubrificantes íntimos, sendo necessário o cuidado do terapeuta para que escolhesse um produto que fosse melhor para a condutividade. ${ }^{14,15}$

Os agentes de acoplamento devem ser levados em consideração, pois são responsáveis pelo contato entre eletrodo e pele, podendo influenciar os resultados da aplicação das correntes. ${ }^{16}$ Eletrodos de silicone-carbono apresentaram menores valores de impedância; ${ }^{12}$ também deve-se observar o prazo de validade do agente e as recomendações de armazenamento. ${ }^{13}$

Para reduzir a impedância, a pele deve ser tricotomizada, que consiste na remoção dos pelos, e limpa com álcool 70\%, para remover eventuais secreções que possam gerar dificuldade na passagem da corrente. Lavando a pele, com água morna, também se reduz a impedância, pois se remove parte da queratina e oleosidade, deixando-a úmida e aquecida. O aquecimento da pele também pode reduzir sua resistência. ${ }^{17}$

Segundo Fuirini Jr. ${ }^{18}$ correntes aplicadas aos tecidos foram influenciadas, tanto pela impedância, quanto pela capacitância. Os sistemas capacitivos tendem a bloquear as correntes contínuas, porém tendem a permitir a passagem de correntes alternadas, sendo que, quanto maior a frequência da corrente alternada, mais facilmente a mesma atravessará o sistema.

Gauglitz $^{19}$ realizou um estudo visando avaliar a agradabilidade das correntes Interferencial (IFC) e estimulação elétrica nervosa transcutânea (TENS) e concluiu que os estimuladores IFC não permitiam diminuição da impedância da pele, quando comparado a TENS. No estudo de Roriz, ${ }^{20}$ sobre tratamento da fibromialgia, foi utilizada a TENS convencional para mapeamento dos tender points, sítios anatômicos específicos dolorosas à palpação, ${ }^{21}$ através da impedância mais baixa no local. Outro estudo ${ }^{22}$ utilizou corrente difásica, durante 5 minutos, antes de outras correntes, visando reduzir a impedância dos tecidos e prepará-los para as correntes seguintes.

As correntes seguem o caminho de menor oposição, portanto a impedância do meio determinará o caminho da corrente, podendo modificar as respostas desencadeadas pelas mesmas. ${ }^{12}$

\section{CONSIDERACִÕES FINAIS}

De acordo com o presente estudo podemos perceber a carência de produções científicas relacionadas aos temas. Assim, recomendamos futuros estudos que investiguem o comportamento da impedância e relutância em recursos eletroterapêuticos, visto a sua grande importância. Podemos perceber que diversos mecanismos estão relacionados à impedância elétrica da pele e sua redução está associada à sensação agradável relatada pelo paciente e a efetividade do tratamento. Também devemos levar em consideração, a importância para o profissional conhecer os assuntos tratados no presente estudo, pois desta forma ele poderá aplicar, de maneira mais efetiva, as correntes excitomotoras, gerando assim uma maior agilidade nos tratamentos fisioterapêuticos.

\section{REFERÊNCIAS}

1. Low J, Red A. Eletroterapia explicada: princípios e prática. São Paulo: Manole; 2001. 472 p.

2. Dingley J, Francis J. Electro Anaesthesia - from torpedo fish to TENS. The Association of Anaesthetists of Great Britain and Ireland 2015;70:93-103. doi: 10.1111/anae.12887

3. Cuypers K, Leenus DJF, Van Den Berg FE, Levin O, Thijs $\mathrm{H}$, Swinnen SP, Meesen RLJ. Long-term TENS treatment decreases cortical motor representation in multiple sclerosis. Neurosciense 2013;10(250):1-7. doi: 10.1016/j. neuroscience.2013.06.046

4. Petra B, Krewer C, Golaszewski S, Koenig E, Müller F. Functional electrical stimulation - assisted active cycling therapeutic effects in patients with heiparesis from 7 days to 6 months after stroke: a randomized controlled pilot study. Arch Phys Med Rehabil 2015;96(2):188-96. doi: 10.1016/j. apmr.2014.09.033

5. Ennis WJ, Lee C, Plummer M, Meneses P. Current status of the use of modalities in wound care: eletrical stimulation and ultrasound therapy. Plast Reconstr Surg 2011;127(Suppl 1): 93-102. doi: 10.1097/PRS.0b013e3181fbe2fd

6. Maan ZN, Januszyk M, Rennert RC, Duscher D, Rodrigues M, Fujiwara T, Whitmore A, Hu MS, Longaker MT, Gurtner GC. Noncontact, Low-Frequency Ultrasound Therapy Enhances Neovascularization and Wouns Healing in Diabetic Mice. Plast Reconstr Surg 2014;134(3):402-11. doi: 10.1097/ PRS.0000000000000467

7. Goode PS, Burgio $\mathrm{KL}$, Johnson TM, Clay OJ, Roth DL, Markland AD, Burkhardt JH, Issa MM, Lloyd LK. Behavioral Therapy With or Without Biofeedback and Pelvic Floor Electrical Stimulation for Persistent Postprostatectomy Incontinence - A Randomized Controlled Trial. JAMA 2011; 
305(2):151-9. doi: 10.1001/jama.2010.1972

8. Valls MGC, Queiroz ES, Meneghetti CHZ, Giusti HHKD. Análise dos efeitos da eletrolipólise no tratamento do fibro edema gelóide por meio da biofotogrametria computadorizada. Fisioterapia Brasil 2012;13(1):54-8.

9. Hooker DN. Correntes de estimulação elétrica. In: Pretince WE. Modalidades terapêuticas para fisioterapeutas. Porto Alegre: Artmed; 2004. p. 77-128

10. Agne JE. Eu sei eletroterapia. Santa Maria: Pallotti, 2009. $399 \mathrm{p}$.

11. Fowler R. Fundamentos da eletricidade: Corrente contínua e magnetismo. Porto Alegre: Bookman; 2013. 256 p.

12. Bolfe VJ, Ribas SI, Montebelo MIL, Guirro RRJ. Comportamento da impedância elétrica dos tecidos biológicos durante a estimulação elétrica transcutânea. Rev Bras Fisioter 2007;11(2):153-9. doi: 10.1590/S141335552007000200011

13. Bolfe VJ, Guirro RRJ. Resistencia elétrica dos géis e líquidos utilizados em eletroterapia no acomplamento eletrodo-pele. Rev Bras Fisioter 2009;13(6):499-505. doi: 10.1590/S141335552009000600006

14. Morais JD, Vasconcelos MS, Lucena EMF, Leite MF, Lyra WS. Resistencia elétrica de géis lubrificantes utilizados na reabilitação uroginecológica. Fisioterapia Brasil 2012;13(6):149-53.

15. Morais JD, Vasconcelos MS, Leite MF, Lucena EMF, Lyra WS. Géis lubrificantes são bons agentes de acoplamento para estimulação transvaginal do assoalho pélvico?. Fisioterapia Brasil 2012;13(6):145-8.
16. Nolan TP. Electrotherapeutic modalities: electrotherapy and iontophoresis. In: Michlovitz SL, Nolan TP. Modalities for therapeutic intervention. Philadelphia: F. A. Davis Company; 2005. p. 97-121.

17. Packer AC, Berni KCS, Bigatin DR. Efeito Da Estimulação Elétrica De Alta Voltagem Sobre A Co-Contração Dos Músculos Mastigatórios Na Disfunção Temporomandibular Um Estudo Piloto. $7^{\text {a }}$ Mostra Acadêmica UNIMEP, 2009.

18. Fuirini Jr N. Parâmetros contemporâneos das correntes excitomotoras. Dissertação de mestrado, apresentada ao Programa de Pós-Graduação em Bioengenharia, da Universidade do Vale do Paraíba. São José dos Campos. São Paulo.

19. Gauglitz ACF, Gomes MCSM, Freitas G, Ribeiro IAC, Silva MD. Comparação do grau de agradabilidade na aplicação da estimulação elétrica nervosa transcutânea e da corrente interferencial. Revista Brasileira de Ciências da Saúde 2005;3(6):18-23. doi: 10.13037/rbcs.vol3n6.454

20. Roriz IMM, Farias MSQ, Barros GG, Câmara TMS, Bastos VPD. Estimulação elétrica nervosa transcutânea na modulação da dor dos tender points na síndrome de fibromialgia: estudo de caso. Rev Saúde.com 2008;4(2):177-89.

21. Helfenstein M, Feldman D. Síndrome da Fibromialgia: características clínicas e associações com outas síndromes disfuncionais. Rev Bras Reumatol 2002;42(1):8-14.

22. Macedo ABC, Cunico F, Sassi L, Albuquerque J, Borges F. Efeitos da aplicação da corrente polarizada e da iontoforese na gordura localizada em mulheres. Fisioter Mov 2013;26(3):65764. doi: 10.1590/S0103-51502013000300020

Como citar: NEPOMUCENO, Patrik; REIS, Claudia Betina; WEIS, Luciana Cezimbra. Influência da Impedância e Relutância em Recursos Eletroterapêuticos Utilizados em Tratamentos Fisioterapêuticos. Cinergis, Santa Cruz do Sul, v. 18, dez. 2017. ISSN 21774005. Disponível em: <https://online.unisc.br/seer/index.php/cinergis/article/view/10460>. Acesso em: 16 jan. 2018. doi:http:// dx.doi.org/10.17058/cinergis. v18i0. 10460. 